\title{
Phase diffusion and charging effects in Josephson junctions
}

\author{
Hermann Grabert \\ Fakultät für Physik, Albert-Ludwigs-Universität, Hermann-Herder-Straße 3, D-79104 Freiburg, Germany \\ Gert-Ludwig Ingold \\ Institut für Physik, Universität Augsburg, Memminger Straße 6, D-86135 Augsburg, Germany \\ Benjamin Paul \\ Fachbereich Physik, Martin-Luther-Universität, Selkestraße 9E, D-06099 Halle, Germany
}

\begin{abstract}
The supercurrent of a Josephson junction is reduced by phase diffusion. For ultrasmall capacitance junctions the current may be further decreased by Coulomb blockade effects. We calculate the Cooper pair current by means of time-dependent perturbation theory to all orders in the Josephson coupling energy and obtain the current-voltage characteristic in closed form in a range of parameters of experimental interest. The results comprehend phase diffusion of the coherent Josephson current in the classical regime as well as the supercurrent peak due to incoherent Cooper pair tunneling in the strong Coulomb blockade regime.
\end{abstract}

74.50.+r, 73.23.Hk, 05.40.+j

New lithography and low-temperature techniques have allowed the fabrication and measurement of small Josephson junctions affected by the capacitive charging energy of single Cooper pairs [1] 3]. Much of the work so far has concentrated on the region of strong Coulomb blockade where the tunneling of Cooper pairs described by the Josephson energy can be treated perturbatively. While Coulomb blockade of Cooper pair tunneling is fairly well understood, the relation between the effects observed at low temperatures and the familiar "classical" dynamics of Josephson junctions 4 remains to be exemplified. In this article we demonstrate that the current peak caused by incoherent Cooper pair tunneling in the regime of strong Coulomb blockade gradually evolves into the classical supercurrent when parameters are changed accordingly.

We consider a Josephson junction with capacitance $C$ and critical current $I_{c}=(2 e / \hbar) E_{J}$, where $E_{J}$ is the Josephson energy. The capacitance gives rise to a charging energy $E_{c}=2 e^{2} / C$ for Cooper pairs. The junction is coupled to an ideal voltage source through a resistor of resistance $R$ as shown in Fig. 1. This system can be modeled by the Hamiltonian

$$
H=H_{J}+H_{\mathrm{env}}
$$

where the first term

$$
H_{J}=-E_{J} \cos (\varphi)
$$

is related to the tunneling of Cooper pairs through the Josephson junction. In analogy to the familiar relation between the voltage across the Josephson junction and the corresponding phase difference

$$
V_{J}=\frac{\hbar}{2 e} \dot{\varphi}
$$

we may introduce a phase

$$
\varphi_{R}=\frac{2 e}{\hbar} V t-\varphi
$$

related to the voltage $V_{R}=V-V_{J}$ across the resistor where $V$ is the external voltage. In the following, we are interested in a voltage regime far below the gap voltage so that tunneling of quasiparticles may be neglected.

The second part of the Hamiltonian (1) describing the coupling of the junction to its electrodynamic environment reads [5]

$$
H_{\mathrm{env}}=\frac{Q^{2}}{2 C}+\sum_{n=1}^{\infty}\left[\frac{q_{n}^{2}}{2 C_{n}}+\left(\frac{\hbar}{2 e}\right)^{2} \frac{1}{2 L_{n}}\left(\varphi_{R}-\varphi_{n}\right)^{2}\right] .
$$

The first term represents the charging energy where the charge $Q$ obeys the commutation relation $[\varphi, Q]=2 i e$. By virtue of Eq. (田), the second part describes a bilinear

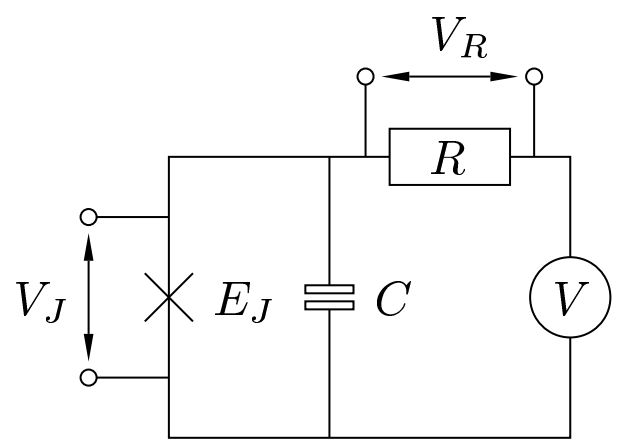

FIG. 1. A Josephson junction characterized by the Josephson energy $E_{J}$ and capacitance $C$ is coupled to a voltage source $V$ via a resistor $R$. The voltage drops across the junction and the resistor are $V_{J}$ and $V_{R}$, respectively. 
coupling between the phase difference across the Josephson junction and phases of a set of $L C$-circuits which model the electromagnetic environment of the junction. The phases $\varphi_{n}$ and charges $q_{n}$ are conjugate variables according to $\left[\varphi_{n}, q_{n}\right]=2 i e$. It can be shown that by suitably choosing the parameters $C_{n}$ and $L_{n}$, the second part of $H_{\text {env }}$ effectively describes the resistor $R$.

The thermal equilibrium Cooper pair current at temperature $T=1 / k_{B} \beta$ may be written as

$$
I_{S}=I_{c}\left\langle\mathcal{U}^{+}\left(\infty, t_{0}\right) \sin \left[\varphi\left(t_{0}\right)\right] \mathcal{U}\left(\infty, t_{0}\right)\right\rangle_{\beta}
$$

where the time dependence of $\varphi(t)$ arises from the Hamiltonian (5).

$$
\mathcal{U}\left(t, t_{0}\right)=\mathcal{T} \exp \left(\frac{i}{\hbar} \int_{t_{0}}^{t} d t^{\prime} E_{J} \cos \left[\varphi\left(t^{\prime}\right)\right]\right)
$$

with the time ordering operator $\mathcal{T}$ is the time evolution operator in the interaction representation. Taking the limit $t \rightarrow \infty$, the precise value of the initial time $t_{0}$ becomes irrelevant and will be set to zero in the following. Expanding the current (6) in powers of the Josephson energy $E_{J}$ one obtains

$$
\begin{aligned}
I_{S}= & i \frac{I_{c}}{2} \sum_{M=1}^{\infty}\left(\frac{i}{2 \hbar} E_{J}\right)^{2 M-1} \sum_{\{\zeta, \eta\}}\left(\prod_{k=1}^{2 M-1} \eta_{k}\right) \zeta_{0} \\
& \times \int_{0}^{\infty} d t_{1} \ldots \int_{0}^{t_{2 M-2}} d t_{2 M-1} \\
& \times \exp \left(i \frac{2 e}{\hbar} V \sum_{k=0}^{2 M-1} \zeta_{k} t_{k}\right) \\
& \times \exp \left(-\sum_{k=1}^{2 M-1} \sum_{l=0}^{k-1} \zeta_{k} \zeta_{l} J\left[\eta_{k}\left(t_{k}-t_{l}\right)\right]\right) .
\end{aligned}
$$

For given order $M$, the $\zeta_{k}, k=0, \ldots, 2 M-1$, which arise from a decomposition of the trigonometric functions in Eqs. (6) and (7) into exponentials, may take the values \pm 1 and are subject to the constraint $\sum_{k=0}^{2 M-1} \zeta_{k}=0$. The factors $\eta_{k}$ also take the values \pm 1 and account for the fact that Eq. (6) contains two time-ordered operators. Finally, the phase correlation function [5]

$$
J(t)=2 \int_{-\infty}^{+\infty} \frac{d \omega}{\omega} \frac{\operatorname{Re} Z_{t}(\omega)}{R_{Q}} \frac{e^{-i \omega t}-1}{1-e^{-\beta \hbar \omega}}
$$

contains the relevant information on the total environmental impedance.

In our case, the impedance as seen from the tunnel junction contains a capacitance $C$ in parallel with a resistance $R$ leading to

$$
\frac{\operatorname{Re} Z_{t}(\omega)}{R_{Q}}=\frac{\rho}{1+\left(\omega / \omega_{R}\right)^{2}} .
$$

The dimensionless impedance at zero frequency is $\rho=$ $R / R_{Q}$ with the resistance quantum $R_{Q}=h / 4 e^{2}$, while the frequency scale is set by $\omega_{R}=1 / R C$.

For the impedance (10), evaluation of the integral (9) gives

$$
\begin{gathered}
J(t)=-2 \rho\left[\frac{\pi}{\hbar \beta}|t|+S+\frac{\pi}{2} e^{-\omega_{R}|t|} \cot \left(\frac{\beta \hbar \omega_{R}}{2}\right)\right. \\
-\sum_{n=1}^{\infty} \frac{e^{-\nu_{n}|t|}}{n\left[1-\left(\nu_{n} / \omega_{R}\right)^{2}\right]} \\
\left.+i \frac{\pi}{2}\left(1-e^{-\omega_{R}|t|}\right) \operatorname{sgn}(t)\right]
\end{gathered}
$$

with

$$
S=\gamma+\frac{\pi^{2} \rho}{\beta E_{c}}+\psi\left(\frac{\beta E_{c}}{2 \pi^{2} \rho}\right) .
$$

Here, $\gamma=0.5772 \ldots$ is Euler's constant and the $\nu_{n}=$ $2 \pi n / \hbar \beta$ are the Matsubara frequencies. Further, $\psi(x)$ is the logarithmic derivative of the gamma function and $\operatorname{sgn}(x)$ denotes the signum function.

Now, typical lead resistances are of the order of $100 \Omega$ [6] so that $\rho \ll 1$. Then, for small Josephson junctions the large lead conductance overdamps the junction and $\omega_{R}$ is large compared to the Josephson frequency

$$
\omega_{J}=\frac{2 e}{\hbar} R I_{c}
$$

Hence, we may disregard terms proportional to $\exp \left(-\omega_{R} t\right)$, and Eq. (11) reduces to

$$
\begin{aligned}
J(t)=-2 \rho[ & \frac{\pi}{\hbar \beta}|t|+S-\sum_{n=1}^{\infty} \frac{e^{-\nu_{n}|t|}}{n\left[1-\left(\nu_{n} / \omega_{R}\right)^{2}\right]} \\
& \left.+i \frac{\pi}{2} \operatorname{sgn}(t)\right] .
\end{aligned}
$$

Furthermore, since $\rho \ll 1$, the ratio $\nu_{1} / \omega_{J}=1 / \rho \beta E_{J}$ may be large even for low temperatures, and the result (14) can be simplified further to read

$$
J(t)=-2 \rho\left[\frac{\pi}{\hbar \beta}|t|+S+i \frac{\pi}{2} \operatorname{sgn}(t)\right] .
$$

Inserting this correlation function into (8), the time integrals may be performed and one obtains the expansion

$$
I_{S}=I_{c} \operatorname{Re}\left(\sum_{M=1}^{\infty} \sum_{\left\{x_{k}\right\}} \prod_{k=1}^{2 M-1} a\left(x_{k}\right)\right),
$$

where the set of positive integers $\left\{x_{k}\right\}$ describes a sequence with $\left|x_{k+1}-x_{k}\right|=1$ starting and ending at $x_{1}=x_{2 M-1}=1$. Each value of $x_{k}$ is associated with a factor

$$
a\left(x_{k}\right)=\frac{\sin \left(\pi \rho x_{k}\right)}{2 \pi \rho x_{k}} \frac{\exp \left[2 \rho S x_{k}\left(x_{k+1}-x_{k}\right)\right]}{\nu+i x_{k} / \beta E_{J}}
$$


which contains the dependence of the Cooper pair current on the applied voltage $V$ via the dimensionless variable $\nu=V / R I_{c}$. An expansion of the form (16) appears in the context of the one-dimensional Coulomb gas [7] and was also found in a previous treatment of the dynamics of small current-biased Josephson junctions [8] for a different kind of approximation. Following [7], we may rewrite (16) in terms of a continued fraction

$$
I_{S}=I_{c} \operatorname{Re}\left[\frac{\sin (\pi \rho)}{2 \pi \rho} \frac{\exp (-2 \rho S)}{\nu+i / \beta E_{J}} \frac{1}{1+\frac{b_{1}}{1+\frac{b_{2}}{1+\ldots}}}\right]
$$

with coefficients

$$
b_{n}=\left(\frac{\beta E_{J}}{2 \pi \rho}\right)^{2} \frac{\sin (\pi \rho n) \sin (\pi \rho(n+1)) \exp (-2 \rho S)}{n(n+1)\left(n-i \nu \beta E_{J}\right)\left(n+1-i \nu \beta E_{J}\right)} .
$$

It is instructive to first investigate the limit $\rho \rightarrow 0$ where the coefficients simplify to read

$$
b_{n}=\left(\frac{\beta E_{J}}{2}\right)^{2} \frac{1}{\left(n-i \nu \beta E_{J}\right)\left(n+1-i \nu \beta E_{J}\right)}
$$

and the continued fraction (18) may be evaluated by means of a matrix recursion [9]. The recursion relations can be solved in terms of modified Bessel functions of complex order. For finite $\rho \ll 1$ the corrections are not necessarily small since in the current-voltage characteristic (18) $\rho$ is multiplied by a factor $S$ defined in Eq. (12) which may contain large terms of order $\log \left(\beta E_{c} / \rho\right)$. On the other hand, a numerical evaluation of the continued fraction converges rapidly and only coefficients $b_{n}$ with moderately large $n$ are relevant. Thus, for $\rho \ll 1$, the sine functions in Eq. (19) may be linearized and it is readily seen that the dominant corrections are described in terms of an effective Josephson energy

$$
E_{J}^{*}=E_{J} \exp (-\rho S)
$$

The continued fraction may then be evaluated by the method described above leading to the current-voltage characteristic

$$
I_{S}=\frac{2 e}{\hbar} E_{J}^{*} \operatorname{Im}\left(\frac{I_{1-i \beta e V / \pi \rho}\left(\beta E_{J}^{*}\right)}{I_{-i \beta e V / \pi \rho}\left(\beta E_{J}^{*}\right)}\right) .
$$

This constitutes the central result of this paper. The dependence of the effective Josephson energy on temperature is depicted in Fig. 2 for various values of $\rho$.

To investigate the classical limit of the current-voltage characteristic (22) one must know that the limit $\hbar \rightarrow 0$

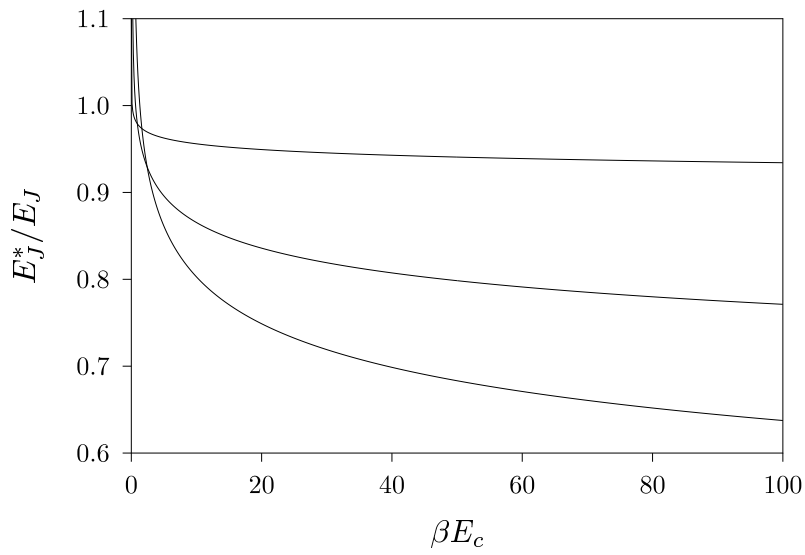

FIG. 2. The effective Josephson energy $E_{J}^{*}$ is shown as a function of the inverse temperature for $\rho=0.01,0.05$, and 0.1 from the upper to the lower curve.

has to be performed such that the flux quantum $h / 2 e$ remains constant. This means that $\rho$ is of order $\hbar$ and $E_{c}$ of order $\hbar^{2}$. Then, in the overdamped limit $\omega_{R} \gg \omega_{J}$ the effective Josephson energy coincides with the bare Josephson energy $E_{J}$. This can also be seen in Fig. 2 where for small $\rho$ the range in which $E_{J}^{*}$ practically equals $E_{J}$ becomes very large. In this case, the current-voltage characteristic 222 can be shown to reduce to the standard Ivanchenko-Zil'berman result [10,11] for classical overdamped Josephson junctions.

While in the classical limit $\beta E_{c} / \rho$ vanishes, in the limit of strong Coulomb blockade we have $\beta E_{c} / \rho \gg 1$. The psi function in Eq. (12) may then be approximated by a logarithm to obtain

$$
S=\gamma+\ln \left(\beta E_{c} / 2 \pi^{2} \rho\right) .
$$

Current-voltage characteristics for overdamped ultrasmall Josephson junctions have so far only been calculated to lowest order in the Josephson energy. The supercurrent arising from incoherent Cooper pair tunneling then reads [11 13 ]

$$
I_{S}=f \frac{|\Gamma(\rho-i \beta e V / \pi)|^{2}}{\Gamma(2 \rho)} \sinh (\beta e V)
$$

where

$$
f=\frac{\pi e}{\hbar} \frac{E_{J}^{2}}{E_{c}} \rho^{2 \rho}\left(\frac{\beta E_{c}}{2 \pi^{2}}\right)^{1-2 \rho} \exp (-2 \rho \gamma) .
$$

In Fig. 3, we compare our result (22) (full line) with the standard Ivanchenko-Zil'berman result (dotted line) and the Coulomb blockade result (24) (dashed line) for $\beta E_{J}=1, \rho=0.04$, and two different values for $\beta E_{c}$. For large $\beta E_{c}$, the current-voltage characteristic (22) is in very good agreement with the Coulomb blockade result (24). With decreasing $\beta E_{c}$, the results (22) and 


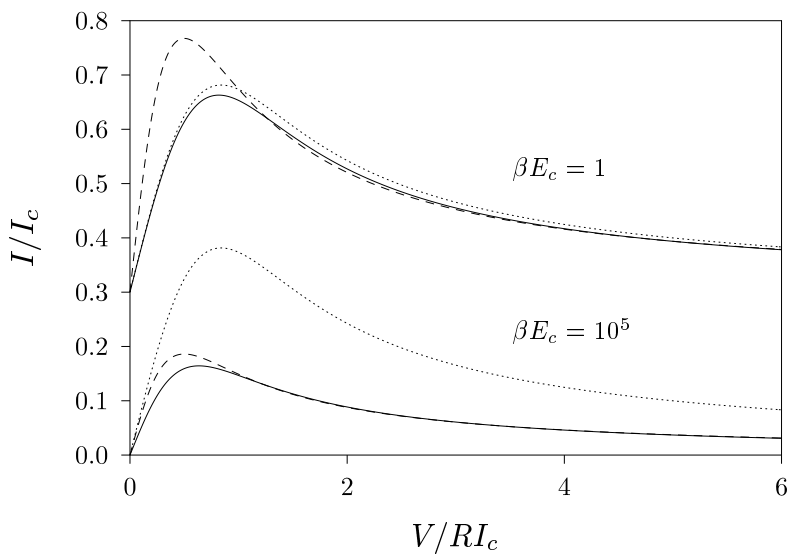

FIG. 3. The current-voltage characteristic of a Josephson junction with Josephson energy $\beta E_{J}=2$ is shown for charging energies $\beta E_{c}=1$ and $10^{5}$ and external resistance $\rho=0.04$. The full line corresponds to our result (22) while the dotted line gives the standard Ivanchenko-Zil'berman result and the dashed line depicts the prediction (24) for strong Coulomb blockade. The two current-voltage characteristics are vertically shifted with respect to each other by $I / I_{c}=0.3$ for sake of clarity.

(24) evolve differently and a crossover to the IvanchenkoZil'berman result can be found.

To make the connection between our result (22) and the Coulomb blockade result (24) more explicit, we consider the current-voltage characteristic (22) to order $E_{J}^{2}$ using the approximation (23). We then obtain for the Cooper pair current

$$
I_{S}=f \frac{2 \pi^{2} \rho \beta e V}{(\beta e V)^{2}+\pi^{2} \rho^{2}} .
$$

While the approximation of the phase correlation function $J(t)$ leading from Eq. (14) to Eq. (15) is only justified for temperatures with $\rho \beta E_{J} \ll 1$, for $E_{J} \ll E_{c}$ the theory should extend to rather low temperatures. In fact, the zero bias differential resistance as well as the voltage for which the current takes its maximum are obtained precisely for small $\rho$ including terms of order $\rho^{2}$.

The result (26) may be improved by going beyond the approximation (15) for the phase correlation function $J(t)$. Keeping the sum over the Matsubara frequencies in Eq. (14), a closed form for $J(t)$ can be found in the strong Coulomb blockade regime $\beta E_{c} / \rho \gg 1$. The current-voltage characteristic (8) may then be evaluated to order $E_{J}^{2}$ and reproduces exactly the expression (24).

In summary, we have derived a formally exact expansion of the current-voltage characteristic of a voltagebiased Josephson junction as a power series in the Joseph- son coupling energy $E_{J}$. Noting that ultrasmall junctions in a standard electromagnetic environment are overdamped, i.e., $\omega_{J} / \omega_{R}=(2 e / \hbar) R^{2} I_{c} C \ll 1$, and with the assumption $\rho \beta E_{J}=\left(e R I_{c} / \pi k_{B} T\right) \ll 1$, the general result was written as continued fraction, which for $\rho \ll 1$ could be summed in closed form. The inferred main result (22) was shown to describe the changeover from the classical Josephson effect in the presence of phase diffusion to the quantum regime of strong Coulomb blockade where Cooper pairs tunnel incoherently. The theory covers the experimentally relevant range for small capacitance Josephson junctions. Although detailed experimental studies of the region between the abovementioned limits are absent, recent work [14] indicates quantum effects in qualitative accord with the predictions made.

We would like to thank the authors of Ref. [14 for stimulating discussions on experimental aspects of the problem and acknowledge helpful conversations with G. Göppert. Financial support was provided by the Deutsche Forschungsgemeinschaft.

[1] G. Schön and A. D. Zaikin, Phys. Rep. 198, 237 (1990).

[2] Single Charge Tunneling, NATO ASI Series B, Vol. 294, ed. by H. Grabert and M. H. Devoret (Plenum, New York, 1991).

[3] M. Tinkham, Introduction to Superconductivity, second edition (McGraw-Hill, New York, 1996).

[4] A. Barone and G. Paternò, Physics and applications of the Josephson effect (Wiley, New York, 1982).

[5] G.-L. Ingold and Yu. V. Nazarov, in Ref. [2].

[6] M. H. Devoret and H. Grabert, in Ref. [2].

[7] A. Lenard, J. Math. Phys. 2, 682 (1961).

[8] W. Zwerger, Phys. Rev. B 35, 4737 (1987).

[9] M. Abramowitz and I. A. Stegun (eds.), Handbook of Mathematical Functions (Dover, New York, 1972).

[10] Yu. M. Ivanchenko and L. A. Zil'berman, Zh. Eksp. Teor. Fiz. 55, 2395 (1968) [Sov. Phys. JETP 28, 1272 (1969)].

[11] G.-L. Ingold, H. Grabert, and U. Eberhardt, Phys. Rev. B 50, 395 (1994).

[12] H. Grabert and G.-L. Ingold, in: Computations for the Nano-Scale, NATO ASI Series E, Vol. 240, ed. by P. E. Blöchl, C. Joachim, and A. J. Fisher (Kluwer, Dordrecht, 1993).

[13] G.-L. Ingold and H. Grabert, Physica B 194-196, 1025 (1994).

[14] D. Vion, M. Götz, P. Joyez, D. Esteve, and M. H. Devoret, Phys. Rev. Lett. 77, 3435 (1996). 